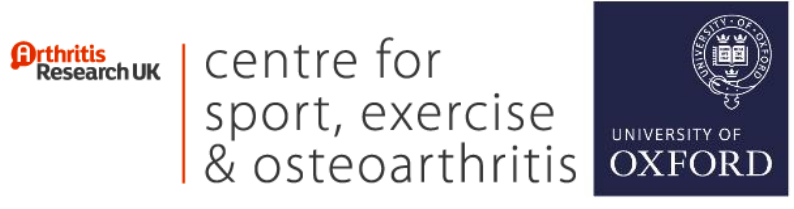

Dr Stephanie Filbay, B.Phty(Hons), PhD

Arthritis Research UK Centre for Sport, Exercise and Osteoarthritis Nuffield Department of Orthopaedics, Rheumatology and Musculoskeletal Sciences, University of Oxford. Windmill Rd. Oxford. UK. OX37LD

\title{
Should surgical repair be recommended over non-operative management for medial meniscus root tears? Letter to the editor
}

\author{
Dear Editor,
}

I read with interest a recent publication in American Journal of Sports Medicine, 'Faucett et al. (2018) Meniscus Root Repair vs Meniscectomy or Nonoperative Management to Prevent Knee Osteoarthritis After Medial Meniscus Root Tears.' ${ }^{1}$ This study undoubtedly addresses a very eminent research topic and makes valuable comparisons between meniscus repair and meniscectomy. However, there is a lack of evidence to support the strong conclusions and clinical recommendations made in relation to non-operative management of medial meniscus root tears, with potential to mislead patients and clinicians.

The message portrayed throughout the results, discussion and conclusion is clear, that repair of medial meniscus root tears compared with non-operative management leads to less osteoarthritis and is a cost saving intervention. However, only 41 of the 355 patients included in the review were treated 'nonoperatively' and these were derived from only 1 study. The study by Krych et al. (2017) was a retrospective review of patients who had not received surgery for a medial meniscus posterior root tear. ${ }^{2}$ The retrospective nature of this study resulted in no standardization of non-operative management. The limited information reported on the non-operative management received indicates that this was not in alignment with current best practice (only $52 \%$ of patients received physical therapy). ${ }^{2}$ Considering the importance and relevance of the type of non-operative management received by the 41 patients in Krych et al. (2017), it is surprising that this is not reported, discussed, or taken into account when drawing such strong conclusions as 'given the current available evidence, medial meniscus root tears should be repaired as the first-line therapy (pg. 5)' and '..posterior root meniscus repair is the clinically and economically superior treatment approach (pg. 7).'

Notably, $51 \%$ of patients who were 'non-operatively' managed had radiographic osteoarthritis (Kellgren and Lawrence $\geq$ grade 2) at baseline. ${ }^{2}$ The purpose of the study by Faucett et al (2018) was to evaluate the longterm effectiveness of three strategies in the management of medial meniscal posterior root tears among those without osteoarthritis. ${ }^{1}$ However, Table 2 reports that $78.8 \%$ of non-operatively managed patients progressed to osteoarthritis. It is unclear where this figure came from since Krych et al. (2017) reported that 'significantly more patients had grade 2 arthritis or higher at final follow-up (78\%) compared to baseline (51\%)' (pg. 385). ${ }^{2}$ This implies that only $27 \%$ of the 41 non-operatively managed patients without osteoarthritis at baseline developed radiographic knee osteoarthritis. It is also worth noting that the only factor associated with an increased rate of arthroplasty in this sample was radiographic osteoarthritis at baseline, and fewer than $10 \%$ of patients without baseline osteoarthritis were estimated to progress to knee arthroplasty within 100 months of injury (fig 3, pg. 386). ${ }^{2}$ Faucett et al. (2018) acknowledged that '..factors such as a high grade of knee osteoarthritis appear to be most predictive of meniscus repair failure' and that this was 'not specifically addressed in our study' (pg. 7). This further questions the strong conclusions made throughout the manuscript regarding non-operative management. 
Several studies were not retrieved during the literature review despite potential to contribute important and relevant information. For example, two studies reported rates of osteoarthritis progression following nonoperative management of a posterior medial meniscus root tear in patients with Kellgren and Lawrence $\leq$ Grade 2 at baseline. ${ }^{3,4}$ In Lim et al (2010), patients received non-steroidal anti-inflammatories for 8-12 weeks and twice weekly supervised physical therapy for 8 weeks. ${ }^{3}$ Only 2 of 30 (7\%) patients (both with Kellgren and Lawrence Grade 2 at baseline) progressed to Kellgren and Lawrence > Grade 2 at a median 36 (range, 24-51) month follow-up. ${ }^{3}$ In Neogi et al (2013), patients received a short course of analgesics and a 12-week supervised exercise program followed by a home exercise program. ${ }^{4}$ Nine of $33(27 \%)$ patients progressed to Kellgren and Lawrence > Grade 2 at a mean 35 (range 26-49) month follow-up. ${ }^{4}$ Clinical measures improved after non-operative management in both studies., 3

Too often systematic reviews draw strong conclusions without demonstrating a sound understanding of the literature from which these conclusions are drawn. This can have critical implications as systematic reviews are ranked the most rigorous in the evidence hierarchy and are typically used by clinicians to guide patient decision making. Researchers who review literature evaluating 'non-operative management' should ensure they critically appraise the management approach in relation to best practice, and take this into careful consideration when interpreting findings and making clinical recommendations.

1. Faucett SC, Geisler BP, Chahla J, et al. Meniscus Root Repair vs Meniscectomy or Nonoperative Management to Prevent Knee Osteoarthritis After Medial Meniscus Root Tears: Clinical and Economic Effectiveness. The American Journal of Sports Medicine.0(0):0363546518755754.

2. Krych AJ, Reardon PJ, Johnson NR, et al. Non-operative management of medial meniscus posterior horn root tears is associated with worsening arthritis and poor clinical outcome at 5-year follow-up. Knee Surg Sports Traumatol Arthrosc. 2017;25(2):383-389.

3. $\operatorname{Lim} \mathrm{HC}$, Bae JH, Wang JH, Seok CW, Kim MK. Non-operative treatment of degenerative posterior root tear of the medial meniscus. Knee Surg Sports Traumatol Arthrosc. 2010;18(4):535-539.

4. Neogi DS, Kumar A, Rijal L, Yadav CS, Jaiman A, Nag HL. Role of nonoperative treatment in managing degenerative tears of the medial meniscus posterior root. J Orthop Traumatol. 2013;14(3):193-199. 